\title{
Persistent Erectile Dysfunction after Discontinuation of 5-Alpha Reductase Inhibitor Therapy in Rats Depending on the Duration of Treatment
}

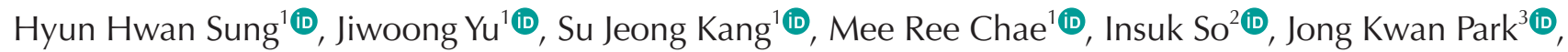 \\ Sung Won Lee ${ }^{1}$ iD \\ ${ }^{1}$ Department of Urology, Samsung Medical Center, Sungkyunkwan University School of Medicine, ${ }^{2}$ Department of Physiology and Biophysics, \\ Seoul National University College of Medicine, Seoul, ${ }^{3}$ Department of Urology, Chonbuk National University College of Medicine and \\ Research Institute of Clinical Medicine of Chonbuk National University-Biomedical Research Institute and Clinical Trial Center of Medical \\ Device of Chonbuk National University, Jeonju, Korea
}

Purpose: The current study is aimed to assess whether a longer duration of $5 \alpha$-reductase inhibitor ( $5 \alpha-$ RI) exposure was associated with higher rate of permanent erectile dysfunction $(E D)$ in a rat model.

Materials and Methods: Male Sprague-Dawley rats $(n=76)$ were assigned to five groups: (i) normal control group; (ii) dutasteride $(0.5 \mathrm{mg} / \mathrm{rat} / \mathrm{d})$ for 4-weeks group; (iii) dutasteride for 4-weeks plus 2-weeks of resting group; (iv) dutasteride for 8-weeks group; and (v) dutasteride for 8-weeks plus 2-weeks of resting group. In vivo erectile responses to electrical stimulation, and changes of fibrotic factors and smooth muscle/collagen contents in the corpus cavernosum were evaluated in each group.

Results: Dutasteride administration for 4 and 8 weeks significantly decreased erectile parameters compared with the control group. Reduced erectile responses were recovered during 2 weeks of drug-free time in the 4-week treatment group, but were not in the 8-week group. Protein levels of fibrosis-related factors transforming growth factor (TGF)- $\beta 1$, TGF- $\beta 2$, and p-Smad/ Smad (Smad 2/3) in the corpus cavernosum showed no significant change after 4 weeks of dutasteride oral administration, but were enhanced after 8 weeks. Dutasteride markedly decreased smooth muscle content and increased collagen after 4 and 8 weeks of use, but no nuclear size changes; however, neither group showed significant improvement in the smooth muscle to collagen ratio after the rest period.

Conclusions: Our study showed that recovery from ED depended on the duration of medication, and administration of dutasteride for more than 8-weeks in rats could result in irreversible ED even after discontinuation of medication.

Keywords: 5-alpha reductase inhibitors; Dutasteride; Erectile dysfunction; Finasteride

This is an Open Access article distributed under the terms of the Creative Commons Attribution Non-Commercial License (http://creativecommons.org/licenses/by-nc/4.0) which permits unrestricted non-commercial use, distribution, and reproduction in any medium, provided the original work is properly cited.

\section{INTRODUCTION}

Benign prostatic hyperplasia $(\mathrm{BPH})$ is a common disease in men over the age of 50 and its incidence increases with age [1]. Treatment of BPH begins with medical therapy, which typically includes 5-alpha re-

Received: Sep 6, 2018 Revised: Nov 8, 2018 Accepted: Nov 13, 2018 Published online Dec 26, 2018

Correspondence to: Sung Won Lee iD https://orcid.org/0000-0001-6608-9050

Department of Urology, Samsung Medical Center, Samsung Biomedical Research Institute, Sungkyunkwan University School of Medicine, 81 Irwon-ro, Gangnam-gu, Seoul 06351, Korea.

Tel: +82-2-3410-3559, Fax: +82-2-3410-3027, E-mail: drswlee@skku.edu 
ductase inhibitors ( $5 \alpha-\mathrm{RIs}$ ) [2]. In fact, less than 1 in 5 men with $\mathrm{BPH}$ is treated with finasteride or dutasteride. Also, many men with $\mathrm{BPH}$ are treated with phosphodiesterase-5 inhibitors. Among an estimated 14 million USA men with symptomatic prostatic hyperplasia, 5a-RIs exposure rose from $4.3 \%$ in 1993 to $15.2 \%$, or 2.1 million men in 2010 [3].

However, in more than $50 \%$ of cases $5 \alpha-\mathrm{RI}$ administration is associated with sexual dysfunction (SD), such as loss of libido, erectile dysfunction (ED), and ejaculatory disorders [4]. Especially in recent years, finasteride-related permanent ED and various symptoms have been reported as post-finasteride syndrome, which collectively could refers to the side effects related to $5 \alpha$ RI medication. Post-finasteride syndrome includes persistent sexual side effects of finasteride in young men [5,6], persistent emotional and cognitive impairment [7], and psychological side effects such as anxious/depressive symptoms and even suicidal thoughts [8,9].

In particular, $5 \alpha$-RIs has been reported to induce ED in animal model and lead to microstructural changes of the corpus cavernosum in a rat model [10,11]. Pinsky et al [10] demonstrated that dutasteride has a detrimental effect on erectile function, and that enhanced inducible nitric oxide synthase (iNOS) expression may protect penile smooth muscle from fibrosis. Zhang et al [11] showed that finasteride exposure for 4 weeks decreased the weight of the penile tissue, although it did not appear to affect the erectile responses to electrical stimulation in a rat model. Furthermore, even if the $5 \alpha-\mathrm{RI}$ is discontinued ED might be persistent and irreversible in the rat model [12].

Two observational studies in humans have shown that longer exposure to finasteride posed a greater risk of ED and worsening ED [13,14]. This study aimed to investigate the effects of dutasteride treatment duration on causing persistent ED even after discontinuation of treatment in a rat model.

\section{MATERIALS AND METHODS}

\section{Ethics statement}

We confirm that this study was conducted with approval from the Institutional Animal Care and Use Committee of Samsung Biomedical Research Institute (No. 20160523001). All experiments were performed in accordance with the relevant guidelines and regulations.

\section{Preparation of animal model}

A total of 76 male Sprague-Dawley rats were used in this study. The rats were assigned to five groups: (i) normal control (n=16); (ii) dutasteride ( $0.5 \mathrm{mg} / \mathrm{rat} / \mathrm{d}$ ) for 4 weeks of oral administration ( $\mathrm{n}=15$ ); (iii) dutasteride for 4 weeks plus 2 weeks of rest (n=15); (iv) dutasteride for 8 weeks of oral administration $(\mathrm{n}=15)$; and (v) dutasteride for 8 weeks plus 2 weeks of rest $(n=15)$. All rats except for those in the normal control group were administered oral dutasteride $0.5 \mathrm{mg} / \mathrm{rat} / \mathrm{d}$. At the time of the experiments, including in vivo cavernous nerve stimulation, western blotting, and histological examination, the ages of all rats were almost equal, ranging from 17 to 20 weeks. In terms of measurement time point (4-week and 8-week), we expected that rats with 4 weeks of dutasteride could be recovered through a 2 -week rest period, but not in the rats with 8 weeks of dutasteride.

\section{Evaluation of in vivo erectile function}

In vivo cavernous nerve stimulation was performed to investigate erectile function. The detailed methodology for measuring the erectile response to cavernous nerve stimulation has been described in previous studies [15]. The electrical field stimulation (EFS) parameters were as follows: $1,2.5$, and $5 \mathrm{~V}, 5 \mathrm{~ms}, 2 \mathrm{~Hz}$, and a duration of $60 \mathrm{~s}$ using an electric stimulator (model PG 721A; Austin Electronic Specialties, Inc., Palo Alto, CA, USA). Sub-maximal EFS was introduced with 1 and $2.5 \mathrm{~V}$ as previously described [15]. By subdividing the power of stimulation, the subtle effects of erection could be compared among the groups. Erectile responses to cavernous nerve stimulation were compared among the five groups under sub-maximal stimulation. At the end of sub-maximal stimulation, the full response was measured using EFS of $5 \mathrm{~V}$ for comparison with sub-maximal stimulation and to ensure that the cavernous nerve was intact. Systemic arterial blood pressure was monitored via carotid artery cannulation with polyethylene-50 tubing. The ratio of maximal intracavernosal pressure (ICP) to mean arterial pressure (MAP) obtained at the peak of the erectile response was determined to control for variations in MAP. In addition, the area under curve (AUC) of ICP/MAP was calculated.

\section{Western blot of the corpus cavernosum}

For Western blot analysis, rat penile tissues stored at $-70^{\circ} \mathrm{C}$ were homogenised in ice-cold protein extrac- 
tion solution (PRO-PREP; iNtRON Biotechnology, Seongnam, Korea) containing 1\% phosphatase inhibitor cocktail 2 and 3 (Sigma Chemical Co., St. Louis, MO, USA) and then centrifuged at 12,000 rpm for $15 \mathrm{~min}$ utes at $4^{\circ} \mathrm{C}$. The protein concentration was determined by Bradford assay (Bio-Rad, Hercules, CA, USA), and equal amounts of protein $(30 \mu \mathrm{g})$ were separated by $4 \%$ to $20 \%$ sodium dodecyl sulfate (SDS)-polyarcylamide gel electrophoresis at $80 \mathrm{~V}$ for 4 hours in Tris/glycine/ SDS buffer (25 mM Tris, $192 \mathrm{mM}$ glycine, 0.1\% SDS, $\mathrm{pH}$ 8.3; Bio-Rad) and transferred onto polyvinylidene difluoride membranes. After transfer, the membranes were blocked with $5 \%$ skim milk powder in Tris Buffered Saline with Tween 20 (TBST) (66 mM Tris base, $137 \mathrm{mM} \mathrm{NaCl}, 2.7 \mathrm{mM} \mathrm{KCl}$, and 0.1\% Tween 20, pH 7.4) for 1 hour at room temperature. The membranes were incubated with primary antibodies at $4^{\circ} \mathrm{C}$ with overnight shaking. Primary antibodies included antiendothelial nitric oxide synthase (eNOS) $(1: 5,000$; BD Biosciences, San Jose, CA, USA), anti-neuronal nitric oxide synthesis (nNOS) (1:200; Santa Cruz Biotechnology, Inc., Dallas, TX, USA), anti-iNOS (1:200; Santa Cruz
Biotechnology, Inc.), anti- $\alpha$-smooth muscle actin (1:1,000; Abcam, Cambridge, UK), anti-transforming growth factor (TGF)- $\beta 1$ (1:500; Abcam), anti-TGF- $\beta 2$ (1:1,000; Abcam), anti-Smad2/3 (1:1,000; Abcam), and anti-p-Smad2/3 (1:1,000; Cell Signaling Technology, Danvers, MA, USA). Anti- $\beta$-actin (1:5,000; Abcam) was used as an internal control. After washing with TBST, the membranes were incubated with secondary antibody (1:2,000; Santa Cruz Biotechnology, Inc.) for 1 hour at room temperature. After washing, signals were detected using a BioRad Laboratories enhanced chemiluminescence detection kit. The generated images were analysed using ImageJ software to determine the integrated density for each protein band.

\section{Masson's trichrome staining of the corpus cavernosum}

The proportion of smooth muscle and collagen in the corpus cavernosum was calculated and compared among the five groups. Masson's trichrome staining was carried out as described previously using a Masson trichrome kit (Abcam) [10,11]. Computer-assisted colour
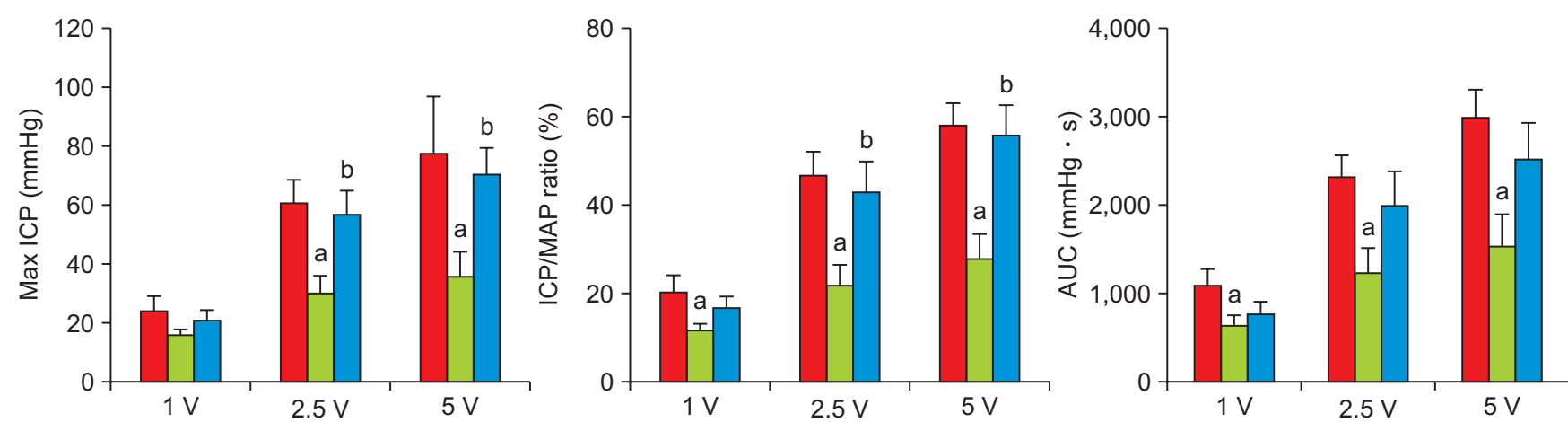

\begin{tabular}{|c|c|c|c|c|}
\hline & & $1 \mathrm{~V}$ & $2.5 \mathrm{~V}$ & $5 \mathrm{~V}$ \\
\hline \multirow{3}{*}{ MBP } & Normal $(n=6)$ & $111.8 \pm 3.5$ & $127.7 \pm 4.5$ & $132.0 \pm 4.4$ \\
\hline & Duta 4 wk $(0.5 \mathrm{mg} / \mathrm{rat} / \mathrm{d})(\mathrm{n}=8)$ & $123.9 \pm 5.5$ & $136.4 \pm 5.6$ & $128.0 \pm 6.2$ \\
\hline & Duta 4 wk+rest 2 wk $(n=8)$ & $121.6 \pm 4.4$ & $132.7 \pm 6.3$ & $125.8 \pm 3.1$ \\
\hline \multirow{3}{*}{ Max ICP } & Normal $(n=6)$ & $24.2 \pm 4.4$ & $60.7 \pm 7.7$ & $77.5 \pm 5.4$ \\
\hline & Duta 4 wk $(n=8)$ & $15.8 \pm 1.3$ & $32.0 \pm 5.1$ & $36.2 \pm 7.4$ \\
\hline & Duta 4 wk+rest 2 wk $(n=8)$ & $20.7 \pm 3.0$ & $56.5 \pm 8.2$ & $70.4 \pm 8.4$ \\
\hline \multirow{3}{*}{ ICP/BP } & Normal $(n=6)$ & $20.6 \pm 3.9$ & $46.9 \pm 5.1$ & $58.1 \pm 5.0$ \\
\hline & Duta 4 wk $(n=8)$ & $12.3 \pm 0.9$ & $22.4 \pm 4.1$ & $28.3 \pm 5.3$ \\
\hline & Duta 4 wk+rest 2 wk $(n=8)$ & $17.1 \pm 2.3$ & $43.3 \pm 6.4$ & $55.9 \pm 6.3$ \\
\hline \multirow{3}{*}{ AUC } & Normal $(n=6)$ & $1,106.9 \pm 165.1$ & $2,313.0 \pm 248.4$ & $3,000.9 \pm 283.7$ \\
\hline & Duta 4 wk $(n=8)$ & $656.6 \pm 81.0$ & $1,234.9 \pm 258.8$ & $2,078.9 \pm 217.0$ \\
\hline & Duta 4 wk+rest $2 w k(n=8)$ & $772.4 \pm 131.7$ & $2,007.6 \pm 368.7$ & $2,547.2 \pm 336.7$ \\
\hline
\end{tabular}

Fig. 1. In vivo erectile responses to serial electrical stimulation in the normal control, 4-week dutasteride group, and 4-week dutasteride with 2-week resting group. Duta: dutasteride, ICP: intracavernosal pressure, MAP: mean arterial pressure, AUC: area under curve. MBP: mean blood pressure, BP: blood pressure. ${ }^{a} \mathrm{p}<0.05$ vs. Normal, ${ }^{b} \mathrm{p}<0.05$ vs. Dutasteride 4 -week group. 
histomorphometry was used to assess the mean percentage of smooth muscle among total erectile tissue (smooth muscle plus connective tissue). Using image analysis software we examined 10 fields ( $\times 100$ magnification) of each tissue section and quantitatively assessed the areas of smooth muscle (stained red) and connective tissues (stained blue) [11].

\section{Drugs and solutions}

Dutasteride was kindly provided by GSK Korea (Seoul, Korea). All other chemical agents were purchased from Sigma Chemical Co. Dutasteride was dissolved in normal saline and administered at $0.5 \mathrm{mg} /$ rat/d via intragastric tube. All other drugs were also dissolved in normal saline.

\section{Data analysis}

Erectile responses were measured with cavernous nerve stimulation and were compared among five groups: normal control, dutasteride 4 weeks, dutasteride 4 weeks plus 2 weeks of rest, dutasteride 8 weeks, dutasteride 8 weeks plus 2 weeks of rest. This compari- son was also performed by serially increasing electrical stimulation voltage. The outcomes of western blot and Masson's trichrome staining were additionally compared among all five groups.

\section{Statistical analysis}

All outcomes were described as the mean \pm standard error. Data were compared using a two-tailed Student t-test and one-way ANOVA test with Bonferroni posthoc analysis. All statistical analyses were conducted using IBM SPSS ver. 23.0 (IBM Co., Armonk, NY, USA), and $\mathrm{p}<0.05$ was considered statistically significant.

\section{RESULTS}

The mean weight of rats was $538.8 \pm 12.3 \mathrm{~g}$ (normal control), $471.7 \pm 8.5 \mathrm{~g}$ (dutasteride for 4 weeks), 504.5 \pm 8.6 g (dutasteride for 4 weeks plus 2 weeks of rest), $560.7 \pm 7.7 \mathrm{~g}$ (dutasteride for 8 weeks), and $540.5 \pm 7.3 \mathrm{~g}$ (dutasteride for 8 weeks plus 2 weeks of rest), respectively $(\mathrm{p}>0.05)$. In the dutasteride experimental groups, no abnormal behaviour was observed during oral ad-
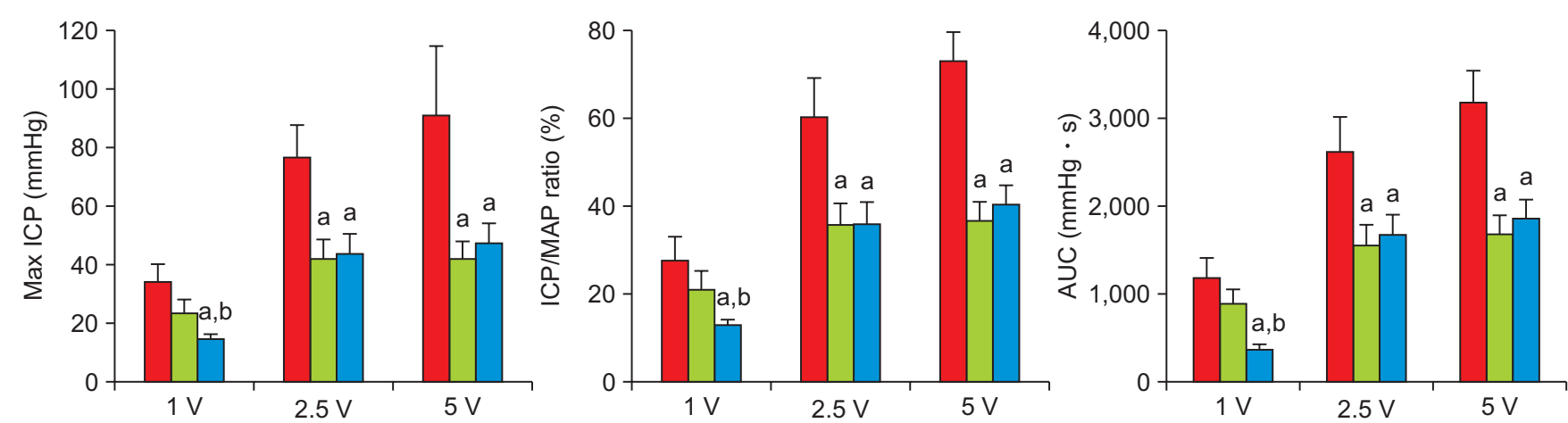

\begin{tabular}{|c|c|c|c|c|}
\hline & & $1 \mathrm{~V}$ & $2.5 \mathrm{~V}$ & $5 \mathrm{~V}$ \\
\hline \multirow{3}{*}{ MBP } & Normal $(n=5)$ & $124.6 \pm 2.5$ & $129.5 \pm 6.9$ & $126.7 \pm 4.3$ \\
\hline & Duta 8 wk $(0.5 \mathrm{mg} / \mathrm{rat} / \mathrm{d})(\mathrm{n}=7)$ & $113.5 \pm 4.4$ & $121.1 \pm 6.3$ & $120.8 \pm 4.6$ \\
\hline & Duta 8 wk+rest 2 wk $(n=7)$ & $115.5 \pm 5.3$ & $119.9 \pm 5.8$ & $118.5 \pm 2.8$ \\
\hline \multirow{3}{*}{ Max ICP } & Normal $(n=5)$ & $35.1 \pm 5.2$ & $77.2 \pm 10.5$ & $91.6 \pm 5.0$ \\
\hline & Duta 8 wk $(n=7)$ & $24.2 \pm 3.9$ & $42.8 \pm 5.8$ & $42.7 \pm 5.1$ \\
\hline & Duta $8 w k+$ rest $2 w k(n=7)$ & $15.2 \pm 0.8$ & $44.2 \pm 6.4$ & $48.2 \pm 5.7$ \\
\hline \multirow{3}{*}{ ICP/BP } & Normal $(n=5)$ & $28.4 \pm 4.7$ & $60.4 \pm 8.6$ & $73.1 \pm 6.1$ \\
\hline & Duta 8 wk $(n=7)$ & $21.7 \pm 3.7$ & $35.9 \pm 4.6$ & $37.0 \pm 4.2$ \\
\hline & Duta $8 w k+$ rest 2 wk $(n=7)$ & $13.3 \pm 0.9$ & $36.4 \pm 4.7$ & $40.4 \pm 4.3$ \\
\hline \multirow{3}{*}{ AUC } & Normal $(n=5)$ & $1,216.4 \pm 201.9$ & $2,631.8 \pm 365.1$ & $3,187.2 \pm 345.7$ \\
\hline & Duta 8 wk $(n=7)$ & $912.9 \pm 148.2$ & $1,581.3 \pm 227.1$ & $1,692.1 \pm 218.0$ \\
\hline & Duta $8 w k+$ rest 2 wk $(n=7)$ & $396.9 \pm 49.2$ & $1,694.6 \pm 219.9$ & $1,887.4 \pm 195.5$ \\
\hline
\end{tabular}

Fig. 2. In vivo erectile responses to serial electrical stimulation in the normal control, 8-week dutasteride group, and 8-week dutasteride with 2-week resting group. Duta: dutasteride, ICP: intracavernosal pressure, MAP: mean arterial pressure, AUC: area under curve. MBP: mean blood pressure, BP: blood pressure. ${ }^{\mathrm{a}} \mathrm{p}<0.05$ vs. Normal, ${ }^{\mathrm{b}} \mathrm{p}<0.05$ vs. Dutasteride 8 -week group 
ministration of dutasteride.

\section{In vivo erectile function of rats}

In vivo erectile responses to cavernous nerve stimulation with $1,2.5$, and $5 \mathrm{~V}$ were increased in a stimulusdependent manner for maximal ICP, ICP/MAP ratio, and AUC of ICP/MAP (Fig. 1). Oral administration of dutasteride for 4 weeks significantly decreased all erectile parameters compared with the control group (all comparisons, $\mathrm{p}<0.05$ ). These reduced erectile responses were almost recovered after 2 weeks of drug-free time (Fig. 1). However, 8 weeks of dutasteride oral administration led to irreversible ED in the rat model (Fig. 2). Rats in both the 8 weeks dutasteride group and the 8 weeks dutasteride plus 2 weeks of rest group showed significantly decreased erectile parameters compared with the control group $(p<0.05)$. In the case of 8 weeks dutasteride group, decreased erectile parameters were not improved by 2 weeks of resting. Representative ICP/MAP traces for each group were shown in the supplement.

\section{Change in fibrotic factors in rat corpus cavernosum induced by dutasteride}

There was no significant change in protein levels of fibrosis-related factors including TGF- $\beta 1$, TGF- $\beta 2$, and p-Smad 2/3 after 4 weeks of dutasteride oral administration ( $p>0.05$; Fig. $3 \mathrm{~A}$ ). In contrast, expression
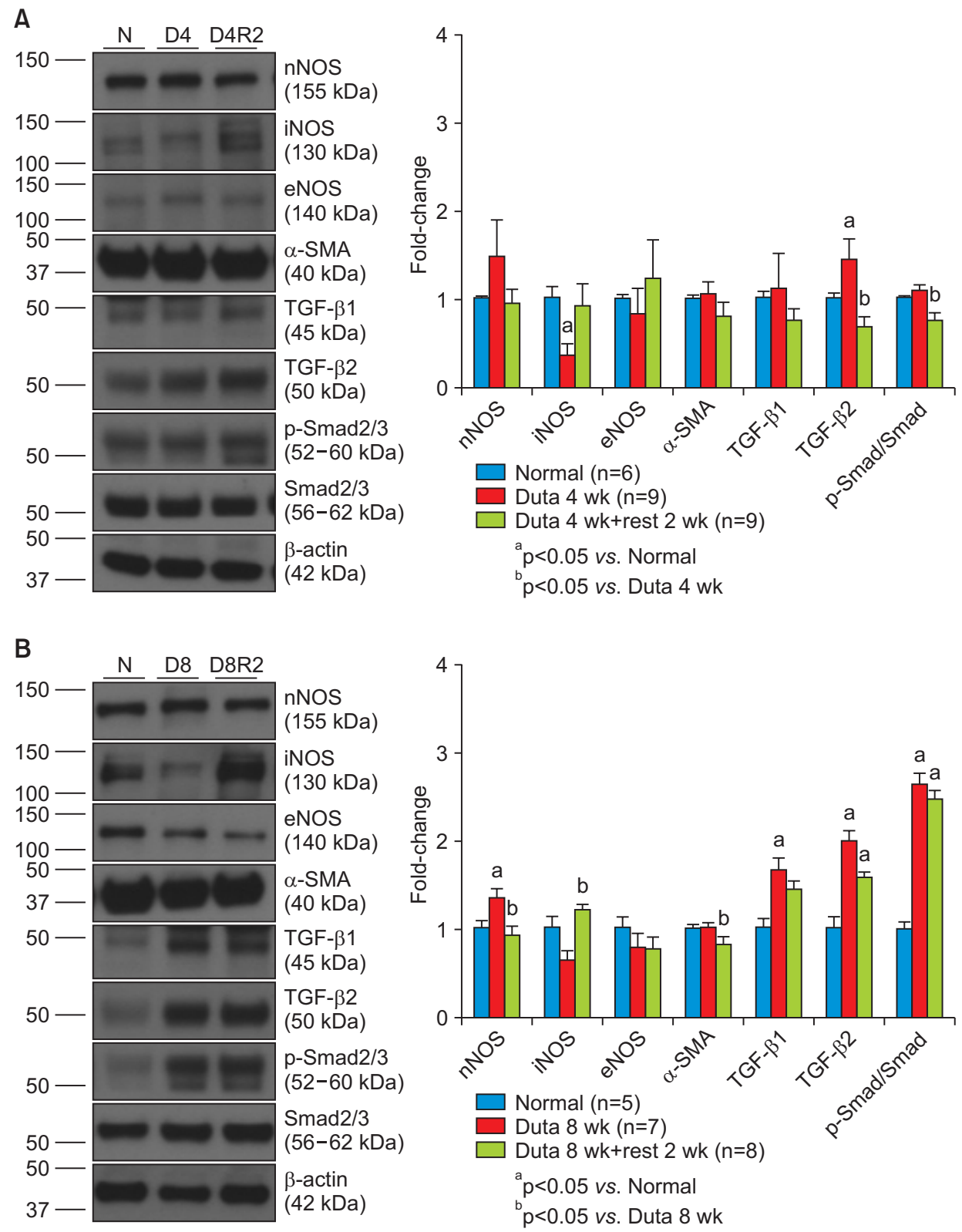

Fig. 3. (A) The changes in protein levels (protein: $40 \mu \mathrm{g}$ ) of fibrosis-related factors transforming growth factor (TGF)- $\beta 1$, TGF- $\beta 2$, and p-Smad/Smad (Smad 2/3) in the 4-week dutasteride group. (B) Changes in protein levels (protein: $40 \mu \mathrm{g}$ ) of fibrosis-related factors in the 8-week dutasteride group. N: normal control, D4: dutasteride 4 weeks, D4R2: dutasteride 4 weeks plus 2 weeks of rest, D8: dutasteride 8 weeks, D8R2: dutasteride 8 weeks plus 2 weeks of rest, nNOS: neuronal nitric oxide synthesis, iNOS: inducible nitric oxide synthase, eNOS: endothelial nitric oxide synthase, SMA: smooth muscle actin, Duta: dutasteride. 

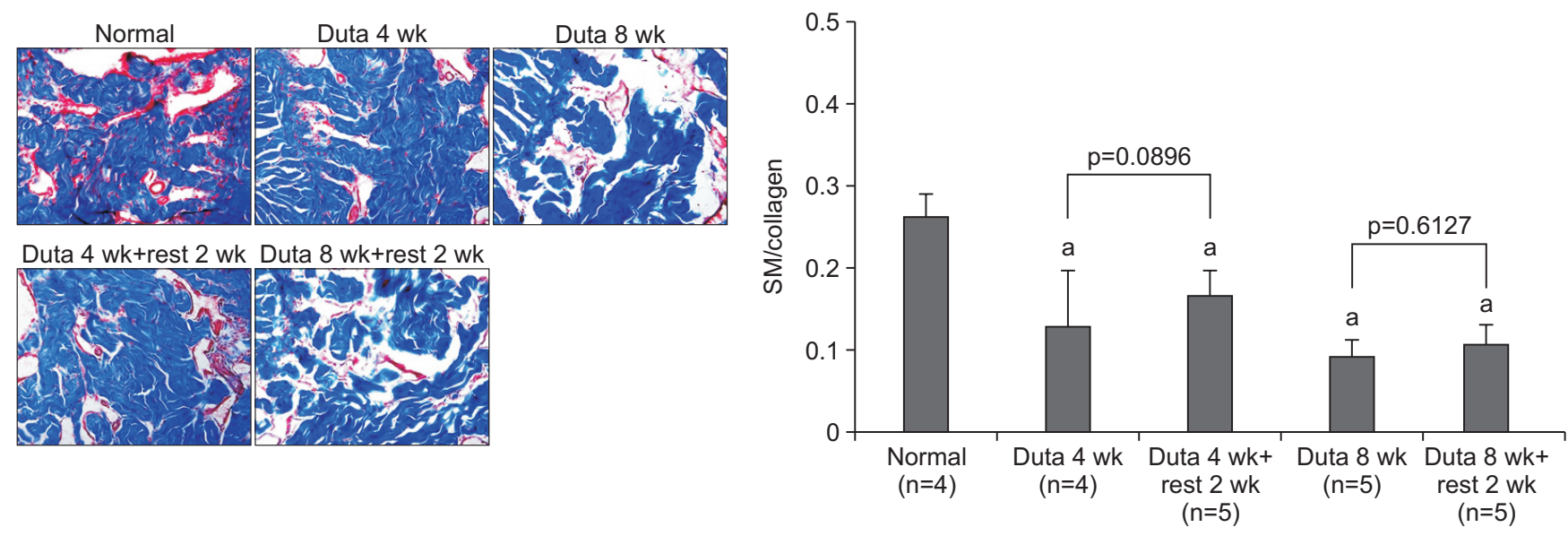

Fig. 4. The changes of immunohistochemical staining of smooth muscle (SM) (staining red) and collagen content (staining blue) in the corpus cavernosum of the rat model (Masson's trichrome staining, $\times 100$ ). Duta: dutasteride. ${ }^{\mathrm{a}} \mathrm{p}<0.05$ compared with normal control.

of TGF- $\beta 1$, TGF- $\beta 2$, and p-Smad 2/3 was significantly enhanced by 8 weeks of treatment with dutasteride ( $<<0.05$; Fig. 3B). Increased levels of these fibrotic factors were maintained even after 2 weeks of resting. There were subtle, but not statistically significant, changes in the level of nNOS and eNOS after medication and discontinuation. A significantly decreased level of iNOS with 4 and 8 weeks of dutasteride administration was observed, and these reductions showed compensatory rebounds over a 2 -week resting period.

\section{Change of smooth muscle contents and fibrosis in rat corpus cavernosum}

The ratio of smooth muscle (staining red) and collagen content (staining blue) in the rat cavernosal tissue was determined using Masson's trichrome staining (Fig. 4). Dutasteride administration markedly decreased smooth muscle content and increased collagen after 4 and 8 weeks of oral administration $(p<0.05)$. There was no significant improvement in the smooth muscle to collagen ratio after the rest period in both the 4- and 8-week groups. In the 4 weeks of dutasteride, it tended to recover microstructural change through 2 weeks of resting, but not significant.

\section{DISCUSSION}

The half-life of dutasteride is known to be 5 weeks in humans [16], so it takes at least 5 to 6 months for washout. In addition, 6 months to 1 year is usually needed for restoration of the structural and functional changes caused by long-term dutasteride treatment in various organs [17,18]. It is assumed that almost 12 days in an adult rat is equivalent to 1 human year using a model based on comparison with normal human lifespan [19]. The half-life of dutasteride is approximately 14 hours in rats [20]. Thus, at least 1 week is necessary for complete wash-out. Taking these aspects into account, we assumed that 2 weeks of rest was a sufficient period to assess the restoration of erectile function after longterm dutasteride treatment in rats, and this was applied in our study as well as in other studies [12].

Long-term dutasteride administration induced a decrease in erectile function response to in vivo nerve stimulation and molecular changes such as a decrease in nNOS and compensatory increase of iNOS, all of which have previously been confirmed in other studies $[10,12]$. iNOS seemed to be suppressed during treatment and then recovered during the rest period. These molecular changes were further enhanced in the rats with 8 weeks of administration compared with 4 weeks. We found that these changes recovered during a 2 -week withdrawal period after 4 weeks of treatment, and not after 8 weeks.

In the present study the increase in collagen and decrease in smooth muscle content at 4 weeks were significantly meaningful. Although sexual functionality was not affected, microstructural changes in the corpus cavernosum were already beginning. In the 4 weeks of dutasteride, it tended to recover microstructural change through 2 weeks of resting, but not significant. It could be explained that the microstructural change did not occur enough to affect the erectile function. In the rat model, long-term suppression of serum dihy- 
drotestosterone (DHT) leads to a decrease in smooth muscle and structural changes, resulting in permanent ED. On the other hand we cannot observe that dutasteride treatment affect nuclear size change of corpus smooth muscle in the current study, although there are reports that androgen deprivation therapy results in small, dense nuclei in prostate cancer [21]. Microstructural changes beyond a critical point eventually affect the function and seem to be irreversible. In this study, we determined the critical point in the rat model to be around 4 to 8 weeks of dutasteride administration. Based on these results, it can be inferred that human beings also have a critical point for $5 \alpha$-RI treatment.

Androgen (including DHT) deficiency is involved in TGF- $\beta /$ Smad signalling pathways that induce fibrosis and increase connective tissue deposition in several organs [22,23]. TGF- $\beta 1$ plays a key role in vascular fibrosis via the Smad pathway [24]. Although it differs in organ and cells, fibrosis shares some common characteristics. However, whether it plays in similar role in the pathological process of penile fibrosis induced by androgen deficiency is not clear. In the current study, the TGF- $\beta /$ Smad pathway was activated in the 8-week dutasteride group and was not recovered by 2 weeks of washout. Experimental results showed that the TGF- $\beta /$ Smad signalling pathway was involved in the pathological process of penile fibrosis caused by 8-week treatment with dutasteride, which might partially explain the underlying mechanism.

These results have several important implications. First, the use of dutasteride medication for longer than a specific period caused a timeframe irreversible ED in the rat model. Duration of administration might be one of the most critical factors in induction of permanent ED in the rat model. Two previous studies demonstrated that $5 \alpha$-RI administration in rats increases the risk of $\mathrm{ED}$ and that some changes in penile smooth muscle function are persistent [10,12]. In addition, the current results showed permanent ED or time-dependent recovery in rats after $5 \alpha$-RI treatment. In addition to the animal model, the present outcome has a critical impact on the clinical setting as permanent ED might also be induced in men treated with long-term $5 \alpha$-RIs with respect to post-finasteride syndrome. We already know the effects of intermittent androgen deprivation therapy on sexual function in patients with prostate cancer [25]. Intermittent therapy has potential benefits with respect to physical function, fatigue, uri- nary problems, hot flashes, libido, and erectile function [26], as well as non-inferiority to continuous androgen deprivation therapy regarding primary oncologic outcomes [25,27]. Extrapolation from studies of intermittent therapy versus continuous therapy leads to the expectation of a beneficial effect of intermittent $5 \alpha$ $\mathrm{RI}$ therapy regarding SD in patients with moderate to severe BPH. It would be clinically meaningful to determine the proper administration duration and recovery time of $5 \alpha$-RIs to maintain the clinical effects on BPH while also reducing adverse events.

Despite the robust data supporting post-finasteride syndrome, little is known about the underlying aetiology driving this phenomenon. A recent meta-analysis supported the view that $5 \alpha$-RIs negatively impact erectile function [28]. Some have speculated that this effect could be nocebo or physiological, resulting from changes in SHBG bound/unbound testosterone in the face of falling DHT levels. Many men have been exposed to these two medications for many years. Recently published data on a large cohort of men who were exposed to finasteride identified that $1.4 \%$ of men had persistent ED after discontinuation, and that this side effect was associated with increased treatment duration [13]. We aimed to identify the factors causing this phenomenon in rats. Our results suggest that duration of $5 \alpha-R I$ medication could be one of the important factors causing $\mathrm{ED}$ in rats.

Actual drug adherence for $\mathrm{BPH}$ has been reported to be considerably low in terms of $5 \alpha$-RI medication with rates of $59 \%$ at 6 months and $18 \%$ at 12 months [29]. One of the most important reasons for this lack of adherence is ED. Even patients with advanced or metastatic prostate cancer still hope that they can have a decent quality of life during treatment of their malignancy. In the case of men with benign disease, such as $\mathrm{BPH}$, preservation of normal sexual function and quality of life during the treatment is essential. It is well known that $5 \alpha$-RIs play beneficial roles in the management of $\mathrm{BPH}$ [30]. Thus, ED in men treated with $5 \alpha$-RIs should be managed carefully.

There are several limitations in the present study. When determining permanent ED of the rat model it is unclear what would happen with a longer wash-out time (i.e., more than 2 weeks). However, as described above 2 weeks of rest should be long enough to evaluate the obvious detrimental effects of $5 \alpha$-RIs on erectile function. Second, we did not figure out more detailed 
time points of dutasteride administration that can cause permanent ED in the rat model. Thus, we could not determine maximal duration of dutasteride without impairment on erectile function. Third, the use of high doses of dutasteride in rats is a limitation. However, the dose was determined by reference to several papers $[10,12]$. In general, it is common to administer high doses of drugs to produce efficacy in animal studies. Lastly, the age of the rats ranged from 17 weeks to 20 weeks and was not constant. This might had some impact on the outcome of erectile function.

\section{CONCLUSIONS}

In a rat model, the reduction of DHT by dutasteride decreased erectile responses to electrical stimulation. This was accompanied by changes in protein levels and histological features, and resulted in increased fibrosis and decreased smooth muscle content in the corpus cavernosum. The current study demonstrated that recovery from $\mathrm{ED}$ depended on the duration of medication, and that administration of dutasteride for longer than a critical period (more than 8 weeks) could result in irreversible $\mathrm{ED}$, even after discontinuation of dutasteride. Further studies on ED and the re-establishment of treatment in human models are essential.

\section{ACKNOWLEDGEMENTS}

This research was supported by a grant of the Korea Health Technology R\&D Project through the Korea Health Industry Development Institute (KHIDI), funded by the Ministry of Health \& Welfare, Republic of Korea (grant number: HI17C0982).

\section{Disclosure}

The authors have no potential conflicts of interest to disclose.

\section{Author Contribution}

Conceptualization: Sung HH, Lee SW. Data curation: Yu J, Kang SJ, Chae MR. Formal analysis: Kang SJ, Chae MR. Funding acquisition: Lee SW. Investigation: Kang SJ, Chae MR. Methodology: Kang SJ, Chae MR, Lee SW. Project administration: Sung HH, Lee SW. Resources: Sung HH, Lee SW. Supervision: SW Lee, So I, Park JK. Writing-original draft: Sung HH, Lee SW. Writing-review \& editing: Lee SW, So I, Park JK.

\section{Supplementary Materials}

Supplementary materials can be found via https://doi.org/ 10.5534/wjmh.180082.

\section{REFERENCES}

1. Berry SJ, Coffey DS, Walsh PC, Ewing LL. The development of human benign prostatic hyperplasia with age. J Urol 1984; 132:474-9.

2. McVary KT, Roehrborn CG, Avins AL, Barry MJ, Bruskewitz RC, Donnell RF, et al. Update on AUA guideline on the management of benign prostatic hyperplasia. J Urol 2011;185: 1793-803.

3. Filson CP, Hollingsworth JM, Clemens JQ, Wei JT. The efficacy and safety of combined therapy with $a$-blockers and anticholinergics for men with benign prostatic hyperplasia: a meta-analysis. J Urol 2013;190:2153-60.

4. Thompson IM, Goodman PJ, Tangen CM, Lucia MS, Miller GJ, Ford LG, et al. The influence of finasteride on the development of prostate cancer. N Engl J Med 2003;349:215-24.

5. Irwig MS, Kolukula S. Persistent sexual side effects of finasteride for male pattern hair loss. J Sex Med 2011;8:1747-53.

6. Irwig MS. Persistent sexual and nonsexual adverse effects of finasteride in younger men. Sex Med Rev 2014;2:24-35.

7. Ganzer CA, Jacobs AR, Iqbal F. Persistent sexual, emotional, and cognitive impairment post-finasteride: a survey of men reporting symptoms. Am J Mens Health 2015;9:222-8.

8. Melcangi RC, Caruso D, Abbiati F, Giatti S, Calabrese D, Piazza F, et al. Neuroactive steroid levels are modified in cerebrospinal fluid and plasma of post-finasteride patients showing persistent sexual side effects and anxious/depressive symptomatology. J Sex Med 2013;10:2598-603.

9. Irwig MS. Depressive symptoms and suicidal thoughts among former users of finasteride with persistent sexual side effects. J Clin Psychiatry 2012;73:1220-3.

10. Pinsky MR, Gur S, Tracey AJ, Harbin A, Hellstrom WJ. The effects of chronic 5-alpha-reductase inhibitor (dutasteride) treatment on rat erectile function. J Sex Med 2011;8:3066-74.

11. Zhang MG, Wu W, Zhang CM, Wang XJ, Gao PJ, Lu YL, et al. Effects of oral finasteride on erectile function in a rat model. J Sex Med 2012;9:1328-36.

12. Oztekin CV, Gur S, Abdulkadir NA, Lokman U, Akdemir AÖ, Cetinkaya $\mathrm{M}$, et al. Incomplete recovery of erectile function in rat after discontinuation of dual 5-alpha reductase inhibitor therapy. J Sex Med 2012;9:1773-81.

13. Kiguradze T, Temps WH, Yarnold PR, Cashy J, Brannigan RE, Nardone B, et al. Persistent erectile dysfunction in men 
exposed to the $5 a$-reductase inhibitors, finasteride, or dutasteride. PeerJ 2017;5:e3020.

14. Traish AM, Haider KS, Doros G, Haider A. Finasteride, not tamsulosin, increases severity of erectile dysfunction and decreases testosterone levels in men with benign prostatic hyperplasia. Horm Mol Biol Clin Investig 2015;23:85-96.

15. Sung HH, Kang SJ, Chae MR, Kim HK, Park JK, Kim CY, et al. Effect of BKCa channel opener LDD175 on erectile function in an in vivo diabetic rat model. J Sex Med 2017;14:5968.

16. Gisleskog PO, Hermann D, Hammarlund-Udenaes M, Karlsson MO. The pharmacokinetic modelling of GI198745 (dutasteride), a compound with parallel linear and nonlinear elimination. Br J Clin Pharmacol 1999;47:53-8.

17. Jeong YB, Kwon KS, Kim SD, Kim HJ. Effect of discontinuation of 5alpha-reductase inhibitors on prostate volume and symptoms in men with $\mathrm{BPH}$ : a prospective study. Urology 2009;73:802-6.

18. Amory JK, Anawalt BD, Matsumoto AM, Page ST, Bremner WJ, Wang C, et al. The effect of 5alpha-reductase inhibition with dutasteride and finasteride on bone mineral density, serum lipoproteins, hemoglobin, prostate specific antigen and sexual function in healthy young men. J Urol 2008;179:23338.

19. Quinn R. Comparing rat's to human's age: how old is my rat in people years? Nutrition 2005;21:775-7.

20. Bramson HN, Hermann D, Batchelor KW, Lee FW, James MK, Frye SV. Unique preclinical characteristics of GG745, a potent dual inhibitor of 5AR. J Pharmacol Exp Ther 1997;282: 1496-502.

21. Dey P. Cancer nucleus: morphology and beyond. Diagn Cytopathol 2010;38:382-90.

22. Traish AM, Park K, Dhir V, Kim NN, Moreland RB, Goldstein I. Effects of castration and androgen replacement on erectile function in a rabbit model. Endocrinology 1999;140:1861-8.
23. Chung CC, Kao YH, Chen YJ, Chen YJ. Androgen modulates cardiac fibrosis contributing to gender differences on heart failure. Aging Male 2013;16:22-7.

24. Ruiz-Ortega M, Rodríguez-Vita J, Sanchez-Lopez E, Carvajal G, Egido J. TGF-beta signaling in vascular fibrosis. Cardiovasc Res 2007;74:196-206.

25. Crook JM, O'Callaghan CJ, Duncan G, Dearnaley DP, Higano CS, Horwitz EM, et al. Intermittent androgen suppression for rising PSA level after radiotherapy. N Engl J Med 2012;367: 895-903.

26. Salonen AJ, Taari K, Ala-Opas M, Viitanen J, Lundstedt S, Tammela TL; FinnProstate Group. Advanced prostate cancer treated with intermittent or continuous androgen deprivation in the randomised FinnProstate study VII: quality of life and adverse effects. Eur Urol 2013;63:111-20.

27. Schulman C, Cornel E, Matveev V, Tammela TL, Schraml J, Bensadoun $\mathrm{H}$, et al. Intermittent versus continuous androgen deprivation therapy in patients with relapsing or locally advanced prostate cancer: a phase $3 \mathrm{~b}$ randomised study (ICELAND). Eur Urol 2016;69:720-7.

28. Corona G, Tirabassi G, Santi D, Maseroli E, Gacci M, Dicuio $M$, et al. Sexual dysfunction in subjects treated with inhibitors of $5 \alpha$-reductase for benign prostatic hyperplasia: a comprehensive review and meta-analysis. Andrology 2017;5:671-8.

29. Cindolo L, Pirozzi L, Fanizza C, Romero M, Tubaro A, Autorino R, et al. Drug adherence and clinical outcomes for patients under pharmacological therapy for lower urinary tract symptoms related to benign prostatic hyperplasia: population-based cohort study. Eur Urol 2015;68:418-25.

30. Roehrborn CG, Siami P, Barkin J, Damião R, Major-Walker $\mathrm{K}$, Nandy I, et al. The effects of combination therapy with dutasteride and tamsulosin on clinical outcomes in men with symptomatic benign prostatic hyperplasia: 4-year results from the CombAT study. Eur Urol 2010;57:123-31. 
150
100
50

50
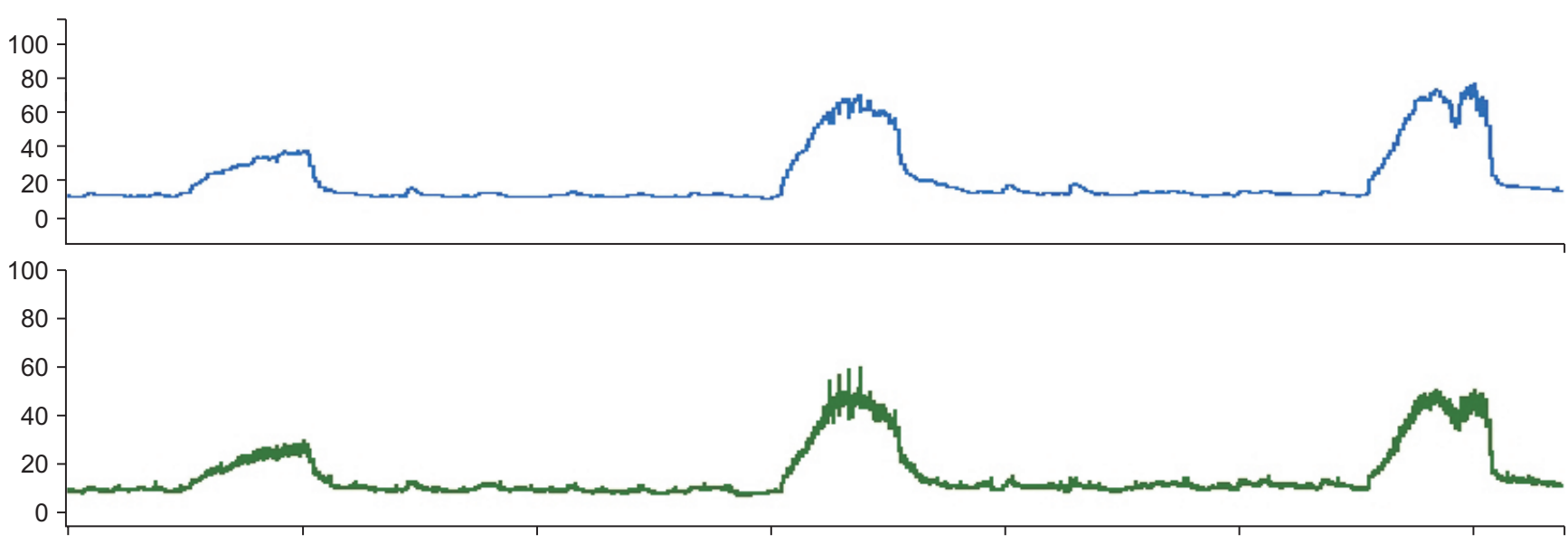

B
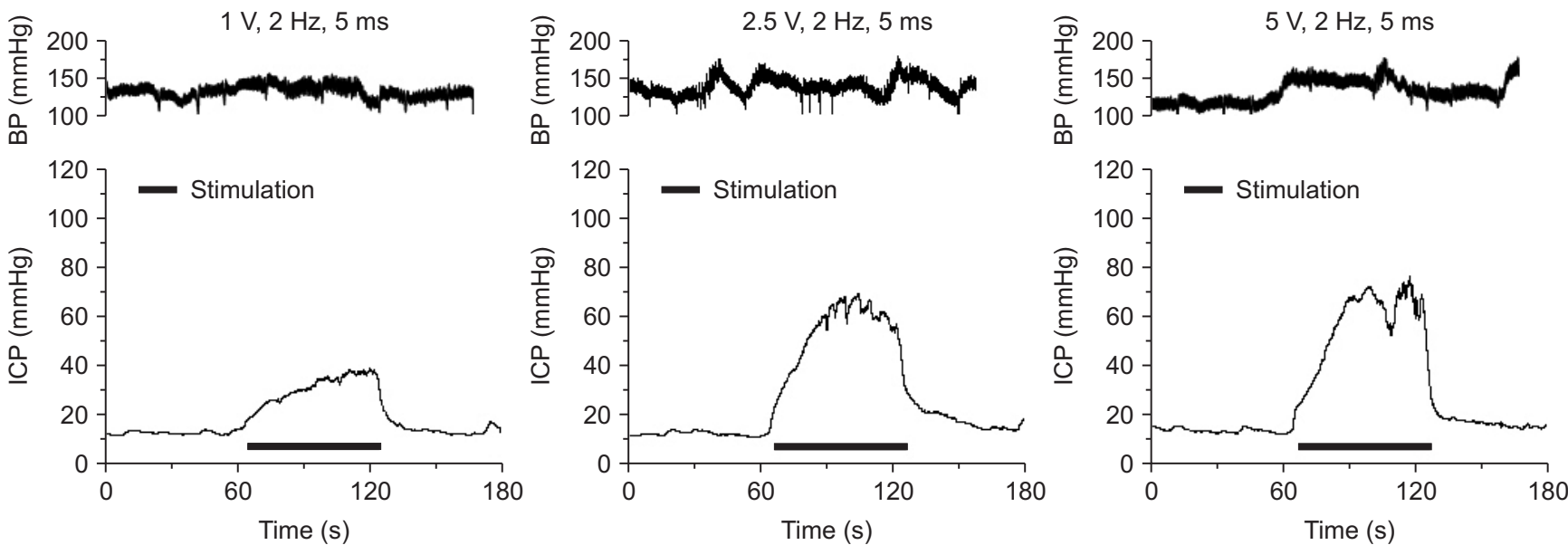

Supplementary Fig. 1. Intracavernosal pressure trace: Normal control 4 wk. 
150

50
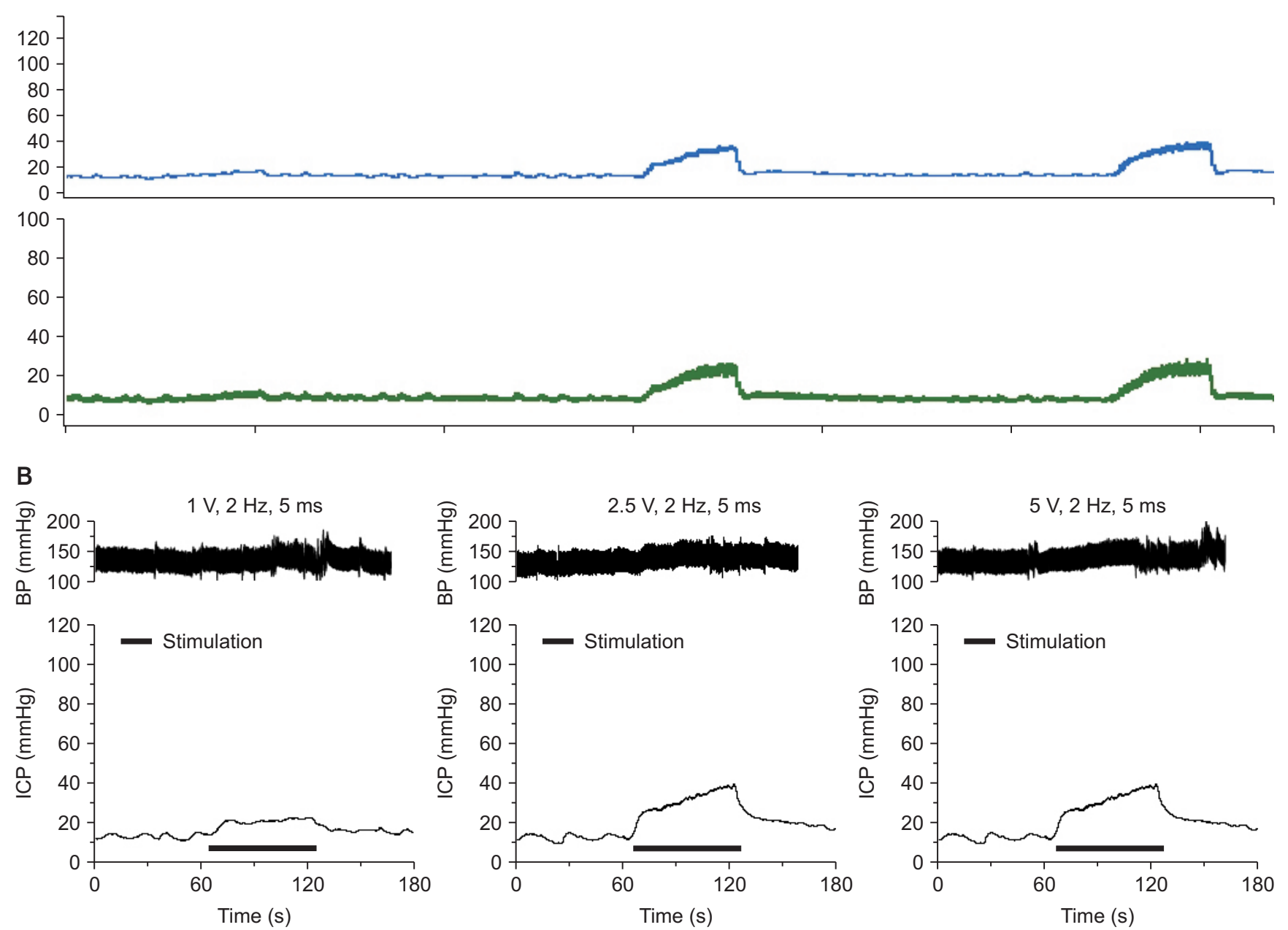

Supplementary Fig. 2. Intracavernosal pressure trace: Duta. control group (Dutasteride 0.5 mg/kg 4 wk P.O). 
150

50
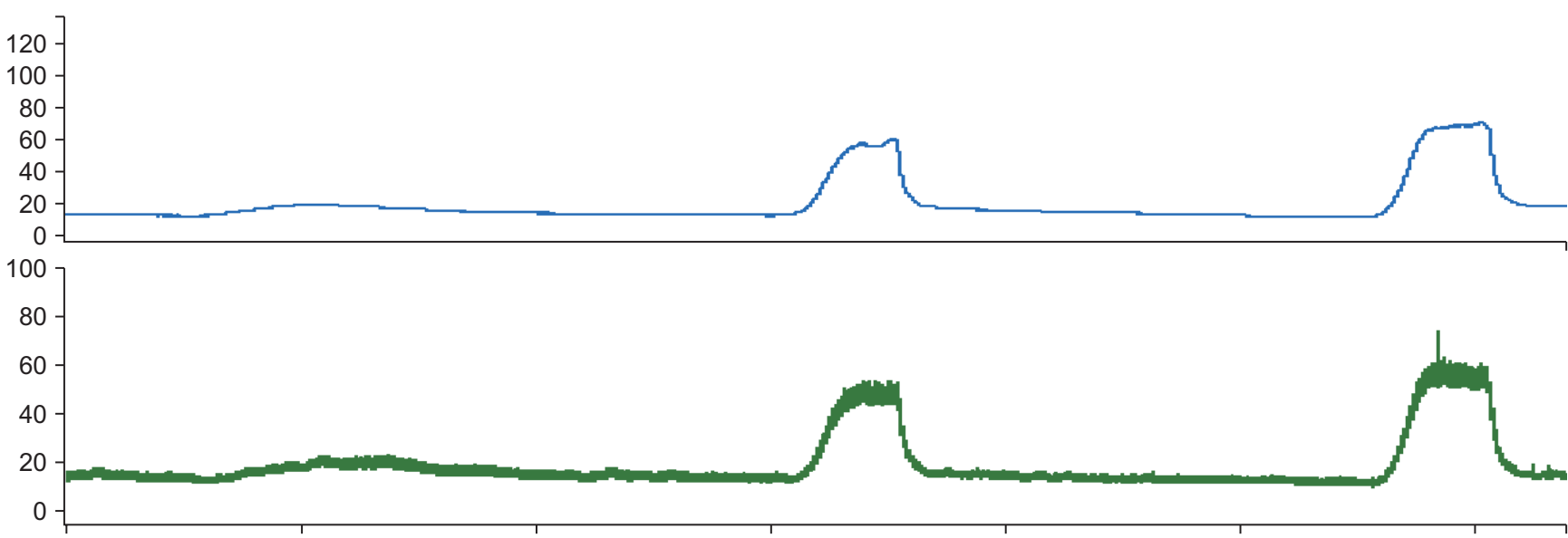

B
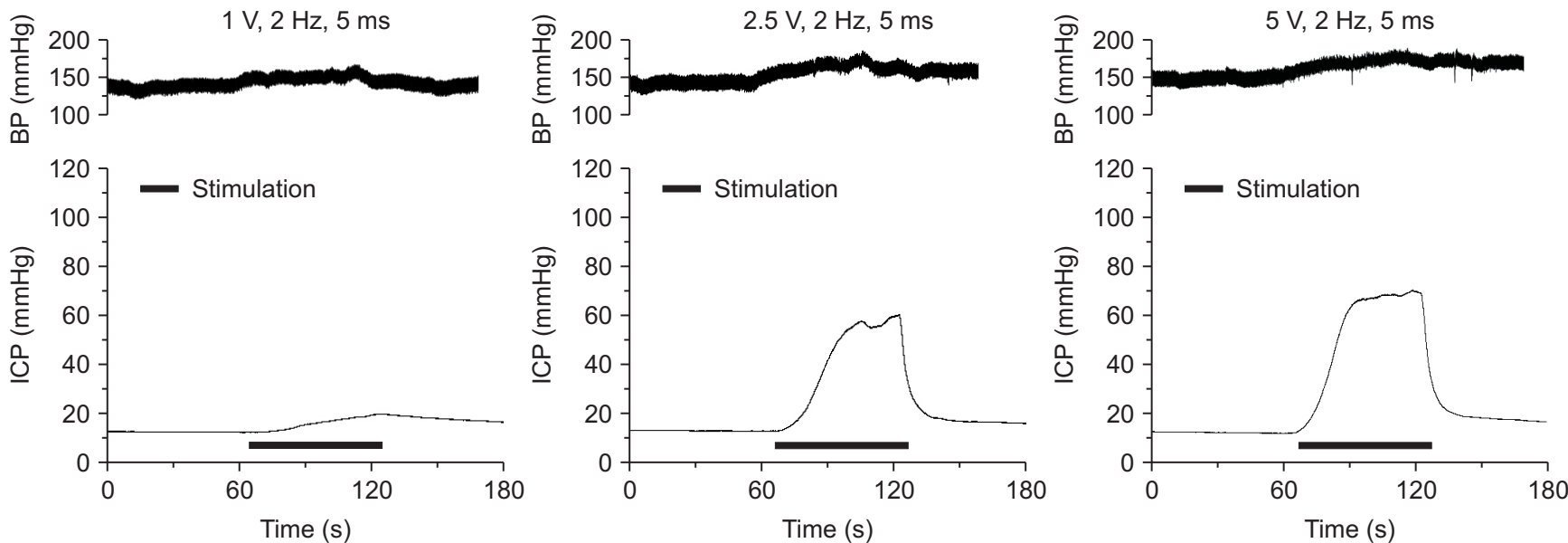

Supplementary Fig. 3. Intracavernosal pressure trace: Normal control 8 wk. 
$150-$

50
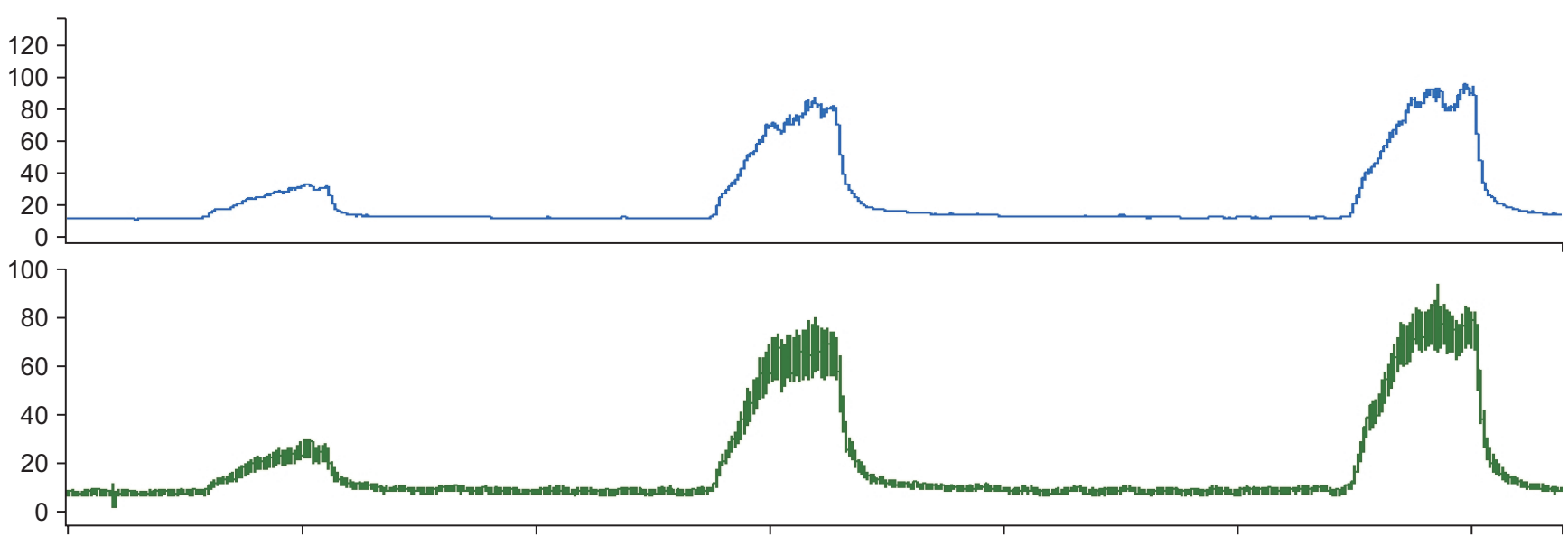

B
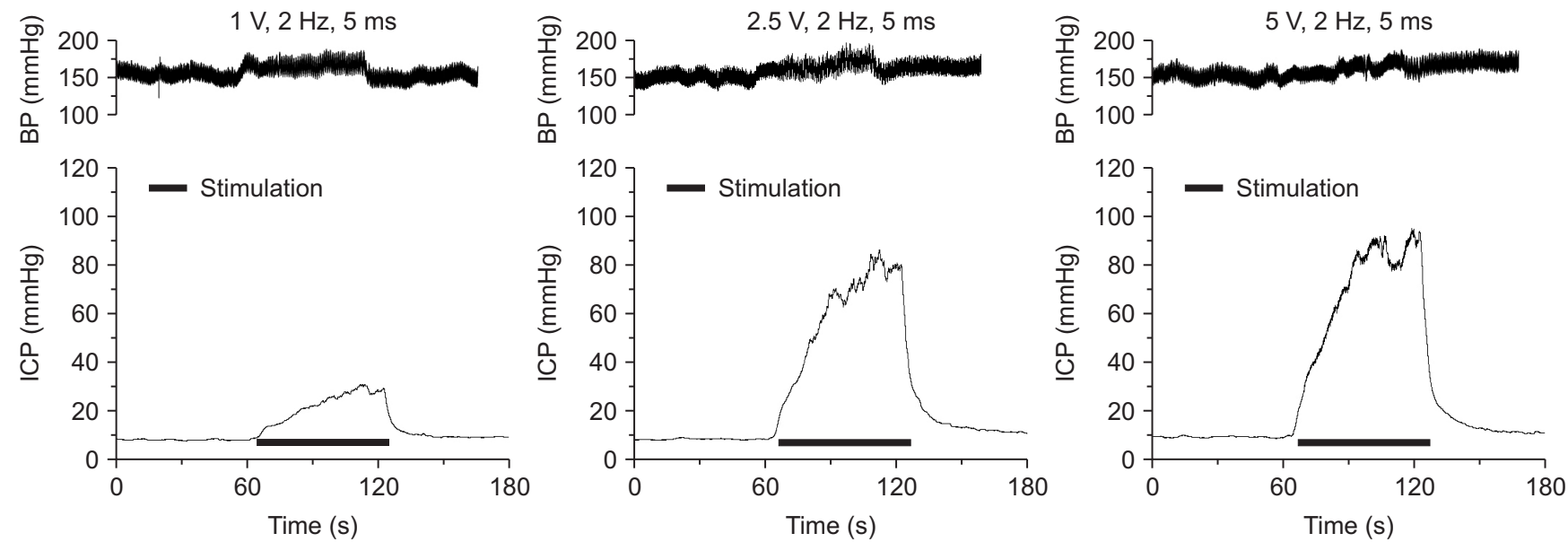

Supplementary Fig. 4. Intracavernosal pressure trace: Normal control 8 wk. 
150

50
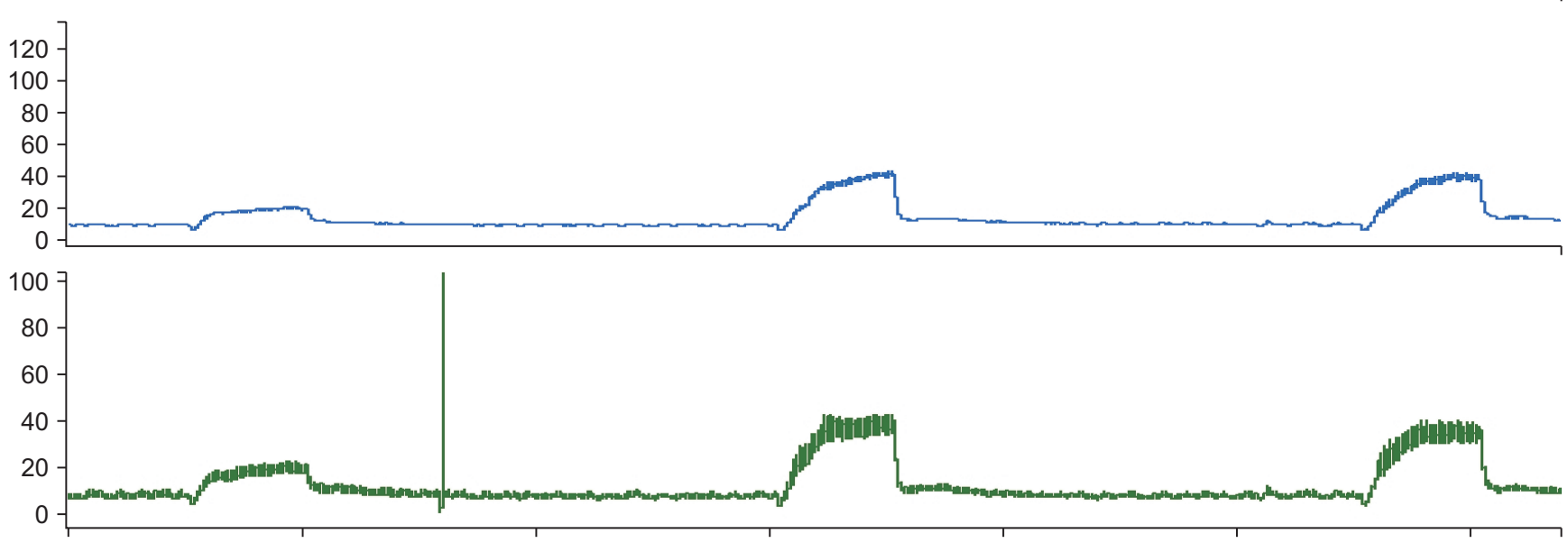

B
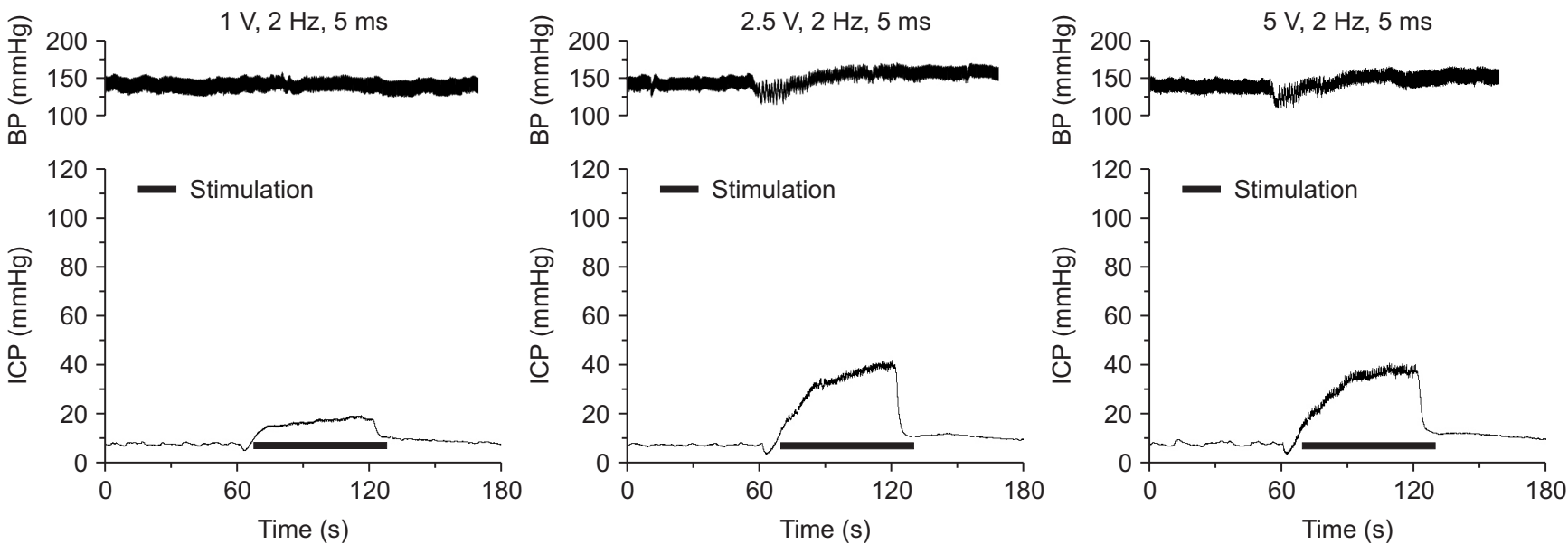

Supplementary Fig. 5. Intracavernosal pressure trace: Duta. control group (Dutasteride 0.5 mg/kg 8 wk P.O) 
150

50
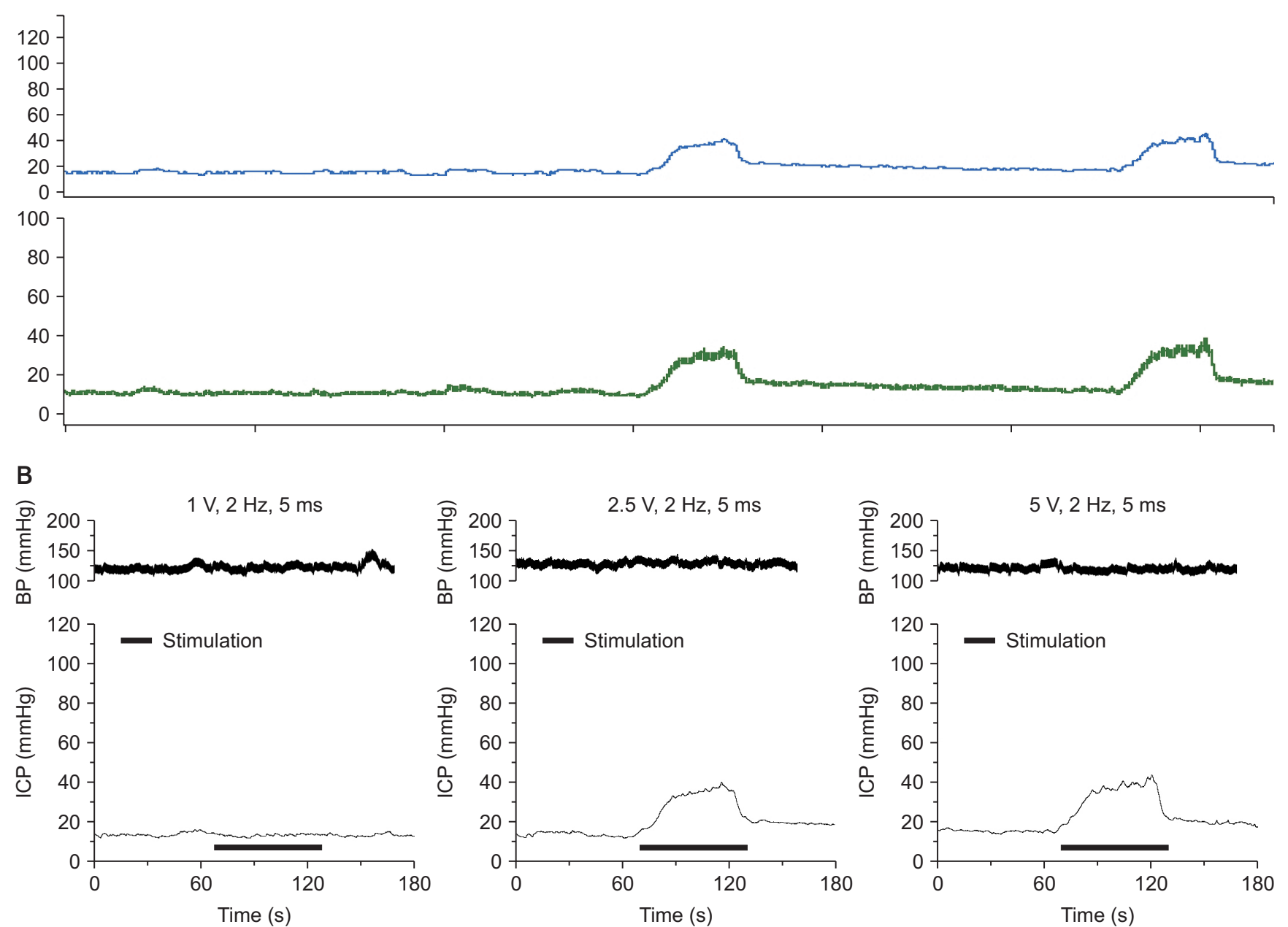

Supplementary Fig. 6. Intracavernosal Pressure Trace: Experimental group (Dutasteride 8 wk P.O+2 wk rest) 